BULL. AUSTRAL. MATH. SOC.

VOL. 33 (1986), 273-278.

\title{
A NOTE ON COMPACT SUBGROUPS \\ OF TOPOLOGICAL GROUPS
}

\section{R.W. Bagley and M.R. Peyrovian}

our main concern is the existence of maximal compact subgroups in a locally compact topological group. If $G$ is a locally compact group such that $P\left(G / G_{0}\right)$, the set of periodic points of $G / G_{0}$, is a compact subgroup of $G / G_{0}$, then $G$ has maximal compact subgroups $K$ such that $G / N$ is a Lie group where $N=n K$, the intersection of the collection $K$ of all maximal compact subgroups of $G$. Also every compact subgroup of $G$ is contained in a maximal compact subgroup. We give an example of a discrete group which has maximal finite subgroups and has finite subgroups not contained in maximal finite subgroups. We note that the above result on $P\left(G / G_{0}\right)$ is an extension of the well-known corresponding result for almost connected groups.

\section{Introduction}

We are interested in the existence of maximal compact and maximal compact normal subgroups of locally compact topological groups. There are two questions. of particular interest: When does a maximal compact normal subgroup exist and is the corresponding factor group a Lie group?

Received 26 June 1985.

Copyright Clearance Centre, Inc. Serial-fee code: 0004-9727/86 $\$ A 2.00+0.00$. 
The other question is, when do maximal compact subgroups exist and when they do, is every compact subgroup contained in a maximal one? It is known that maximal compact normal subgroups do exist in cases for which the corresponding factor groups are not Lie groups [5]. We give an example of a discrete group which has maximal finite subgroups and has finite subgroups not contained in maximal finite subgroups. We also give necessary and sufficient conditions for the existence of maximal compact subgroups $K$ of $G$ such that $K G_{0}$ is normal in $G$ (Theorem 1). One case is that $P\left(G / G_{0}\right)$, the set of periodic elements of $G / G_{0}$, is a compact subgroup. In this case $G / N$ is a Lie group where $N$ is the intersection of the collection of all the maximal compact subgroups, and every compact subgroup of $G$ is contained in a maximal one. This is an extension of the well known corresponding result for almost connected groups, that is, groups $G$ such that $G / G_{0}$ is compact.

Apparently Lemma 1 below is well-known. We include it here for easy reference.

LEMMA 1. If $G$ is a Zocally compact topological group such that $G / G$ 。 is compact, then $G$ contains a collection of maximal compact subgroups $K$ such that $G=K G_{0}$ for each $K \in K$, the elements of $K$ are conjugates of each other, every compact subgroup is contained in an element of $K$ and $G / N$ is a Lie group where $N=n K$, the maximal compact normal subgroup of $G$.

Proof. Since $G / G_{0}$ is compact we can choose a compact normal subgroup $L$ of $G$ such that $G / L$ is a Lie group, [3, p.175]. All the conclusions of the lemma now follow from [4, Theorem 3.1] and [2, p.180].

LEMMA 2. If $G$ is a locally compact topological group and $K$ is a maximal compact subgroup of $G$, then $K G_{0} / G_{0}$ is a maximal compact subgroup of $G / G_{0}$.

Proof. Let $M / G_{0}$ be a compact subgroup of $G / G_{\text {。 }}$ such that $K G$ 。 $\subset M$. Since $K$ is maximal compact in $M$, by Lemma 1 we have $K G$ 。 $=M$. 
LEMMA 3. If $K$ is a maximal compact subgroup of the locally compact topological group $G$, then $K G$ 。 is open in $G$.

Proof. By Lemma 2, $K G_{0} / G_{0}$ is maximal compact in $G / G_{0}$. Let $U$ be a compact neighbourhood of the identity of $G / G_{0}$ and let $V$ be a neighbourhood of the identity such that $x V x^{-1} \subset U$ for each $x \in K G_{0} / G_{0}$. Since $G / G_{0}$ is totally disconnected, there is a compact open subgroup $L_{1} \subset V$. Let $L$ be the subgroup generated by $U\left\{x L_{1} x^{-1}: x \in K G_{0} / G_{0}\right\}$. It follows that $L\left(K G_{0} / G_{0}\right)$ is a compact subgroup of $G / G_{0}$ and $L \subset K G_{0} / G_{0}$. Thus $K G_{0} / G_{0}$ is open in $G / G_{0}$ since $L$ is. This completes the proof that $K G$ o is open in $G$.

THEOREM 1. If $G$ is a locally compact topological group, then the following are equivalent:

I. $P\left(G / G_{0}\right)$ is a compact subgroup of $G / G_{0}$.

II. $G$ has a maximal compact subgroup $K$ such that $K G$ o is normal in G.

III. If $K \neq \phi$ consists of the maximal compact subgroups of $G$, then $K G_{0}=M$ for some subgroup $M$ of $G$ and each $K \in K$. In this situation the following hold:

(i) $M$ is an open normal subgroup of $G$.

(ii) If $K_{1}, K_{2}$ are in $K$, then $m K_{1} m^{-1}=K_{2}$ for some $m \in M$.

(iii) The subgroup $N=n K$ is the maximal compact normal subgroup of $G$ and $G / N$ is a Lie group.

(iv) Every compact subgroup of $G$ is contained in a maximal compact subgroup of $G$.

Proof. To show that I implies II we let $P\left(G / G_{0}\right)=M / G_{0}$, a compact subgroup of $G / G_{0}$. By Lenma $1, M$ has a maximal compact subgroup $K$ such that $M=K G_{0}$. Since $M / G_{0}$ is the maximal compact normal subgroup of $G / G_{0}$, it follows that II holds. If II holds, then by Lemma $2 K G_{0} / G_{0}$ is maximal compact in $G / G_{0}$ if $K$ is maximal compact in $G$. 
Again by Lemma 1 , letting $K$ be the collection of all maximal compact subgroups of $K G_{0}=M$ hence of $G$, we have III. We complete the proof of the equivalence by showing that III implies $I$. It follows from III that $M / G_{0}$ is uniquely the maximal compact subgroup of $G / G_{0}$. Thus $M / G_{0}=P\left(G / G_{0}\right)$

To prove the remainder of the theorem we note that (i) follows from Lemma 3, (ii) from Lemma 1 , and (iii) from Lemmas 1 and 3 . To prove (iv) it is sufficient to show that every compact subgroup of $G$ is contained in $M$ and again appeal to Lemma 1 . Let $L$ be a compact subgroup of $G$ and suppose $x \in L$. Then $x G_{0} \in P\left(G / G_{0}\right)=M / G_{0}$. It follows that $x \in M$ since $M$ is a subgroup containing $G_{0}$. This completes the proof of the theorem.

In contrast to Theorem 1 we have the following.

THEOREM 2. If $G$ is a Zocally compact topological group then every compact subgroup of $G$ is contained in a maximal compact subgroup of $G$ if and only if every compact subgroup of $G / G$ 。 is contained in a maximal one. There exist topological groups with maximal compact subgroups and compact subgroups which are not contained in any maximal one.

Proof. If $M / G_{0}$ is a compact subgroup of $G / G_{0}$, let $M=K G$ 。 where $K$ is a compact subgroup of $G$. If $K$ is contained in a maximal compact subgroup of $G$, then by Lemma $2 M / G_{0}$ is contained in a maximal compact subgroup of $G / G_{0}$. On the other hand if $L$ is a compact subgroup of $G$ and $L G_{0} / G_{0}$ is contained in a maximal compact subgroup $M / G$ of $G / G_{0}$, then $L$ is contained in a compact subgroup maximal in $M$ (hence in G) by Lemma 1. This proves the first part of the theorem. We prove the second part by the following:

Example. Let $G$ be the free product of the two element group $z_{2}=\{1, z\}$ and $X=\sum_{i=1}^{\infty} z^{i}$, the direct sum, where $z^{i}=z_{2}$ for each $i$. $G$ has maximal finite subgroups and has finite subgroups which are 
not contained in a maximal finite subgroup. The proof is contained in the following.

1. If $y=x_{1} z \ldots x_{n} z$ with $x_{1} \neq 1$, then $y$ has infinite order.

Proof. The lemma follows immediately from the fact that every power of $y$ is of the same form as $y$.

2. The subgroup $Z_{2}$ of $G$ is maximal finite.

Proof. If $G_{1}$ is a subgroup properly containing $Z_{2}$, then clearly $G_{1}$ contains an element of the form of $y$ in Lemma 1 .

3. If $G_{1}$ is a subgroup of $G$ which contains a nontrivial subgroup of $X$ and some element not in $X$, then $G_{1}$ contains an element of infinite order.

Proof. If the element in $G_{1}$ not in $X$ is of the form $x_{1} z \ldots x_{n} z, z x_{1} z \ldots z$, or $z x_{1} \ldots z x_{n}$, then clearly $G_{1}$ contains an element of infinite order. If the element in question is of the form $x_{1} z \ldots z x_{n}$, then $x_{1}^{-1} G_{1} x_{1}$ contains an element of one of the three other forms and the conclusion follows in this case also, completing the proof.

COROLLARY. If $G$ has a compact normat subgroup $K$ such that $G / K$ is locally connected, then every compact subgroup of $G$ is contained in a maximal compact subgroup of $G$ if and only if every finite subgroup of $G / K G_{0}$ is contained in a maximal finite subgroup of $G / K G_{0}$.

Proof. By Theorem 1.4[1] $K G_{0}$ is open in $G$. Applying the first part of Theorem 2 to $G / K$ we have every compact subgroup of $G / K$ contained in a maximal compact subgroup if and only if the corresponding condition on finite subgroups of $G / K G$ 。 holds. Since every compact subgroup of $G$ is contained in a maximal one if and only if that same condition holds for $G / K$, the proof is complete.

The following questions come to mind. If $G$ has compact subgroups which are not contained in maximal compact subgroups does $G$ have a totally disconnected subgroup which has a compact subgroup not contained 
in any maximal one? Under what conditions, other than the cases covered by Theorem 1 , is $G / N$ a Lie group if $N=n K$, the intersection of all maximal compact subgroups $K \neq \phi$ of $G$ ? There are examples for which $G / N$ is not Lie and $N=n K$ [5].

\section{References}

[1] R. W. Bagley, T. S. Wu and J. S. Yang, "Localily compact groups in general settings", (submitted).

[2] G. Hochschild, The Structure of Lie groups, (San Francisco 1965).

[3] D. Montgomery and L. Zippin, Topological transformation groups, (Interscience, New York, London 1955.)

[4] G. D. Mostow, "Self-adjoint groups", Ann. of Math. 62 (1955), 44-55.

[5] M. R. Peyrovian, "Maximal compact normal subgroups", Proc. Amer. Math. Soc. (to appear).

Department of Mathematics

University of Miami

Coral Gables

Florida 